\title{
The Effects of Pain Neuroscience Education with Conventional Physical Therapy on Chronic Plantar Fasciitis: A Case Study
}

\author{
Yahya Alfaifi ${ }^{*}$, Dana Webb ${ }^{2}$ \\ ${ }^{1}$ The Physical Therapy Department, The First Medical Center, Riyadh, Saudi Arabia \\ ${ }^{2}$ School of Physical Therapy \& Rehabilitation Sciences, Morsani College of Medicine, University of South Florida, Tampa, USA \\ Email: *albasheqqq@gmail.com
}

How to cite this paper: Alfaifi, Y. and Webb, D. (2021) The Effects of Pain Neuroscience Education with Conventional Physical Therapy on Chronic Plantar Fasciitis: A Case Study. Open Journal of Therapy and Rehabilitation, 9, 57-69.

https://doi.org/10.4236/ojtr.2021.92005

Received: April 10, 2021

Accepted: May 23, 2021

Published: May 26, 2021

Copyright $\odot 2021$ by author(s) and Scientific Research Publishing Inc. This work is licensed under the Creative Commons Attribution International License (CC BY 4.0).

http://creativecommons.org/licenses/by/4.0/

\begin{abstract}
Background and Purpose: Plantar Fasciitis (PF) is a condition that affects the foot and causes complaints such as pain and stiffness. The most common symptom of this condition is pain. There are many treatment options to deal with this condition, such as conservative therapy, medications, and surgical interventions in rare cases. This case study aims to investigate the potential impact of pain neuroscience education (PNE), combined with a conventional Physical Therapy (PT) program on a 37-year-old-patient with chronic plantar fasciitis. Case description: A 37-year-old male health care professional presented to an outpatient physical therapy clinic with a diagnosis of chronic PF for around two years. The participant had tried several treatment options with no improvement of his symptoms. After undergoing a physical therapy evaluation, he was given a plan of care for twelve sessions by a skilled physical therapist for a six-week period, with each session consisting of 30 minutes of conventional PT, followed by 5 to 15 minutes of PNE. Results: After completing the prescribed plan of care, the patient reported a reduction in subjective symptoms via the Visual Analog Scale (VAS). He also reported improvement with symptoms and functional independence via the Foot Function Index (FFI). The patient reported no change in the quality of sleep via Pittsburg Sleep Quality Index (PSQI). Lastly, he demonstrated no objective improvement in foot pressure with the Navicular Drop Test. Discussion: This case report indicates that PNE, combined with conventional PT for PF can have a positive impact on subjective pain and foot function. PNE should involve many topics about the physiology of pain and the nervous system and should be administered by a certified therapeutic pain specialist. Further studies are recommended to investigate the impact of this intervention in combination with traditional PT for PF in larger populations.
\end{abstract}




\section{Keywords}

Neuroscience, Pain, Physical Therapy, Chronic Plantar Fasciitis, Case Study

\section{Introduction}

Plantar fasciitis (PF) is one of the most common conditions of the foot, causing many symptoms such as heel pain [1]. The plantar fascia is composed of elastic collagen fibers that connect the inner part of the foot and extend longitudinally to maintain the integrity of the medial arch [2]. These layers of fibers are called the plantar fascia, and these longitudinal fibers may suffer different types of injuries, such as tears or degeneration [3]. This condition is called plantar fasciitis [3]. This mechanical disturbance leads to an increase in the tensile force on the fascial tissue and changes in the normal distribution of forces around the foot tissues [4]. The etiology of this condition is still not exactly known, but many studies show the connection between the weakness of internal muscles of the foot and the different deformities that affect the high point of the longitudinal arch, such as pes cavus and pes cavum [5]. PF represents around 15\% of foot conditions [6]. Several factors may be related to the development of PF, with the rate increasing for those over 50 years of age [7]. There is a significant association between high body mass index and the risk of developing heel pain due to the increase of pressure areas during loading activities [8]. For example, increased foot plantar flexion is another risk factor for developing PF [4]. Prolonged weight-bearing activities such as running may also lead to PF, with almost $10 \%$ of runners developing PF [5].

The symptoms of this condition may include pain, stiffness, and warmth [5] [9]. Patients often report functional limitations such as difficulty walking, especially in the early morning, sleep disturbance, and an antalgic gait pattern [9].

There are many options for treating PF, including conservative therapy, Extracorporeal Shock Wave Therapy (ESWT), pharmaceutical agents, and in limited cases, surgical intervention [10]. Based on the Clinical Practice Guidelines (CPG) of the American Physical Therapy Association (APTA), every intervention for heel pain has a certain level of evidence regarding its ability to treat PF [9]. For example, stretching exercises for the fascia and plantar flexor muscles possess strong evidence as effective treatments to reduce heel pain, especially in the acute and subacute phases of PF [9] [10].

Soft tissue mobilization for the tissues of the lower limbs is another conservative therapy that is able to reduce the symptoms of PF. However, this therapy should be combined with self-mobilization and stretching as part of a home exercise program [9].

Taping is another intervention that could reduce pain in the acute phase of PF, although it has limited effects on a functional level. This intervention can be more efficient if it is combined with the taping of the plantar flexors and stret- 
ching exercises [9] [11].

Foot orthotics that used as supports for the medial arch of the foot and the heel, are effective treatments to reduce symptoms of PF [9]. The effect of this treatment can be increased by supplementing it with night splints [9]. Another intervention that may be used for PF cases with mild effects includes ESWT, which is based on the CPGs from the APTA in 2014 [9]. This treatment method can be effective in breaking down the adhesions and fibrosis among fascial tissues and relieving pain especially in chronic cases [12] [13].

Another treatment option would be to utilize pharmaceutical agents such as non-steroid anti-inflammatory drugs which aim to relieve pain and decrease inflammation [5]. Corticosteroids, for example, dexamethasone is also an option and can be delivered via iontophoresis, despite the conflicting evidence for its effectiveness [1] [9]. In very rare cases, the "less than one-tenth of PF cases" surgical approach becomes the best choice to reduce symptoms [4]. However, the prognosis for this condition differs between patients, and depends on their individual symptoms and risk factors. It can take a long time to recover, although most cases become asymptomatic in one year [9] [13].

In general, women are at a higher risk of developing PF than men and face worse prognoses [10]. Runners, as well as those with an elevated body mass are also at risk of developing PF [5] [9] [14]. In addition, standing on a rough surface, or walking for a prolonged period of time are also risk factors of PF [9]. Heel pain causes many bothersome symptoms that represent a significant challenge to both patients and therapists due to the usual course of the disease. There is a need to explore alternative treatment methods that could be added to the conventional management of PF. Therapeutic pain education (TNE) is a promising intervention in terms of dealing with chronic pain conditions [15].

TNE opened the doors for Pain Neuroscience Education (PNE), as TNE failed to adequately reduce pain, and was unable to provide patient education in general terms. However, the studies on TNE were not comprehensive and did not include the biological aspect of pain physiology [15].

Some later studies have shown the effectiveness of PNE in treating a variety of diseases and conditions [15] [16]. For example, the intervention usually works to address pain, function, and psychological status [15] [17].

The use of PNE as a therapeutic intervention can help calm the brain, which is part of interoperation for the nociceptors coming from different body parts [15] [16]. A few studies done by Louw and his colleagues show that PNE is an effective treatment method, and that its efficacy dramatically increases when it is used with other treatments, such as manual therapy, neurodynamic mobilization, or therapeutic exercises [15] [16]. Currently, there is no evidence about the potential effects of PNE on chronic pain in patients with PF. Therefore, the purpose of this case report is to highlight and describe the effects of PNE in combination with conventional physical therapy interventions on a 37-year-old patient with chronic PF. 


\section{Case Description}

This report was conducted after receiving permission from the Medical Director and research committee at the First Medical Center. Prior to the initial evaluation, a short lecture was delivered to the patient about the study and the type of interventions that he would receive. He agreed to voluntarily participate in this study and signed a consent form. The patient also is signed consent to give access to his medical records to use them in this report.

A history was taken from the patient, and all available medical information was reviewed. The participant was a 37-year-old male healthcare professional. He was mildly overweight based on his body mass index $\left(27.4 \mathrm{Kg} / \mathrm{m}^{2}\right)$. He was diagnosed with chronic bilateral PF over two years ago (Figure 1).

His symptoms started with pain in the medial aspect of his left foot, and over the next few months progressed to his right foot. However, his left foot remained the most symptomatic. He reported utilizing many pharmaceutical agents to reduce his symptoms but stated that the effects were short-lived. He also reported that he underwent 12 sessions of physical therapy at a private clinic, which still did not alleviate his symptoms. He was referred to our department by an orthopedic physician at our medical center. He stated that he used to be an active individual, reporting he used to walk 5 kilometers. He reported spending most of his time between work and watching movies. The patient was overweight based on his BMI, which was another risk factor of PF [7] [8].

The medical history showed normal health status without any serious illnesses. He was under the care of psychology due to a history of insomnia. There was no history of previous injuries of the lower limbs or back. The patient had undergone an MRI that supported the medical diagnosis of PF. He reported that he used to take some supplements to improve his quality of sleep such as melatonin and magnesium once daily before going to sleep (Table 1).

\section{Examination and Evaluation}

The patient presented to the clinic wearing supportive sports shoes with silicon insole pads. He stated that he believed that these pads were the only treatment option that could reduce his pain, and that he began wearing these shoes 4

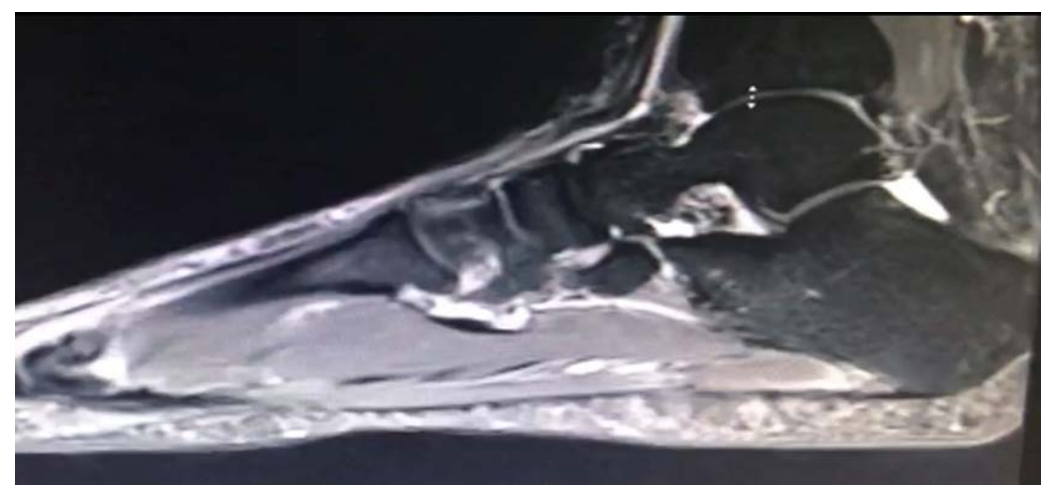

Figure 1. Thickening of the plantar fascia on MRI sagittal view. 
Table 1. Demographic information of the participant.

\begin{tabular}{cc}
\hline Demographic Variable & Value \\
\hline Age & 37 years \\
Sex & Male \\
Body Mass Index & $27.4 \mathrm{Kg} / \mathrm{m}^{2}$ \\
Smoking & Heavy 31.5 pack-years. \\
Educational Level & College \\
Marital Status & Married (lives alone) \\
Alcohol & None \\
Current Lifestyle & Sedentary "due to pain" \\
\hline
\end{tabular}

months prior to this evaluation. The main complaint of the patient was moderate to severe pain in the heel region of the left foot and mild pain in the same region of the right foot. He described his symptoms as "intermittent" and "sharp". According to the visual analog scale (VAS) where 10 is the most pain experienced and 0 is no pain, he reported 5/10 in the left foot after ambulating from his car to the healthcare clinic, which was less than 50 meters. Symptoms exacerbated with prolonged weight bearing activities, such as standing at work and his first step of bed in the morning.

The patient presented with an unknowing antalgic gait pattern. The diagnosis of this case based on the International Classification of Disease (ICD) was plantar fasciitis based on the medical diagnosis and the PT evaluation. The patient reported that his pain increased with prolonged weight bearing activities. During palpation, there were tender points at the medial calcaneal tuberosity, the medial longitudinal arch, and the center of the calcaneus on both feet. There was also muscle weakness in his lower limbs. The gluteal and abductor muscles of the patient were tight and weak, especially on the left side, which could be due to the patient's sedentary work and lifestyle. The muscle strength was tested and graded from 1 to 5 on the Manual Muscle Test Scale (MMT), which is a valid and reliable test to measure muscle strength, where 0 is no muscle contraction and 5 is muscular contraction against gravity with maximum resistance [18]. The grades were 4-/5 hip flexors, knee extensors, knee flexors and 3+ for plantar flexors gastrocnemii and solei, which were tested from prone position bilaterally.

The Windlass Test was performed, which has excellent specificity and poor sensitivity, and was found to be positive bilaterally [19]. The Navicular Drop Test, which is a reliable test to determine the degree of pronation of the foot, was also performed; a decreased 10 millimeters of height difference between the navicular tuberosity and ground in weight bearing and non-weight bearing positions is also a risk factor for developing PF [20]. There was a significant height drop of the left foot, with 13 millimeters for the left foot and 11 millimeters for the right foot. Upon examination of the soft tissue of the lower limbs, a mild contracture of the bilateral medial and lateral parts of gastrocnemius muscles 
and left lateral part of hamstring were noted. This assessment was done by a Silverskoid Test to measure the length of the gastrocnemius muscles during knee flexion and extension [21].

Standardized outcome measures were also assessed. The functional status of the feet was evaluated using the foot functional index (FFI). This is a self-administered questionnaire that examines pain, disabilities, and activity level 23 items. The FFI is valid to measure the functions of the lower limbs [22], with a total possible score of 230 [22].

In this study, the digital reviewed version of the FFI was used [23]. This version is called the Italian Foot Function Index [24]. It covers the same components as the regular FFI, but it is only 17 items with a total possible score of 170 points [24]. The Pittsburgh Sleep Quality Index (PSQI) was also assessed due to the patient reporting that he had insomnia caused by foot pain; this assessment measures the quality of sleep [25].

This outcome measurement is for patients who show sleep disturbance related to chronic pain [25]. All of the tests were repeated before and after the interventions; the FFI was done every time using the digital version, while the PSQI was applied at the baseline treatment and after the last session.

\section{Intervention}

The PT program was selected based on the internal protocol for PF treatment in our department. This protocol involved four main interventions. The first, ESWT, is an effective treatment for PF that provides pain relief and helps to progress rehabilitation programs and improve foot functions [12]. The relief provided by ESWT is even greater than that provided by local injection by cortisone [26]. Manual therapy is the second part of the intervention, and there are many manual techniques used to improve the flexibility of the soft tissues around the foot and the lower limb and to increase the range of motion of the ankle and foot [9] [27]. Brantingham et al. (2009) published a systematic review regarding the effectiveness of manual therapy for lower limb disorders [27]. Their results support the effectiveness of manual therapy in the management of lower limb conditions, especially in the short period after treatment [27]. Therefore, many manual techniques were used to improve the flexibility of soft tissue and reduce spastic tissues; for example, myofascial release techniques are an effective treatment approach in heel pain cases [28]. The mobilization grade III and VI were used for 5 minutes every session to the following foot joints: subtalar, transverse tarsal, cuboideonavicular, tarsometatarsal, intermetatarsal, metatarsophalangeal, and interphalangeal joints [9]. Low dye taping was used as the third intervention for this case [11]. It was used as an alternative treatment method to decrease pressure on the longitudinal medial arch [11]. The fourth intervention was stretching exercises for the lower limbs. Stretching exercises are the cornerstone of PF management [9].

Every session began by stretching the muscles of the lower limbs with a focus 
on the plantar fascia and plantar flexors. These static stretches were performed in seated and standing positions with a thirty-second hold, for five repetitions each.

Strengthening exercises were also included in the treatment plan of this case due to the muscle weakness in the lower limbs. The program involved all of the lower extremity muscles by doing three sets with 15 repetitions for two muscles every session. Hip strengthening exercises were added to the program at week four, due to the opinion of a better prognosis, which could be a key point in PF treatment [28].

This treatment program is almost identical to the one used with our regular PF clients, and the average time of treatment before discharge is 12 sessions. Table 2 shows the therapeutic interventions with this case.

Around $77 \%$ of patients discharge with reduction of their symptoms; the remaining clients are either referred to further treatment options such as corticosteroid injection therapy, or they left the treatment program. For this reason, I added a new intervention, PNE, to this case. PNE was applied for five to fifteen minutes within the plan of treatment, as it is a relatively new and effective method of intervention to reduce chronic pain [16]. This education included general and specific information about the physiology of pain; it also included information on nociceptors transference from different body parts, as well as perception and interpretation of these signals in normal and abnormal situations [17]. This education should involve basic information (Figure 2). During the PNE, the focus was on the biological aspects of pain and on providing comprehensive knowledge about the nervous system's physiology and the factors that alter the normal integration between its parts and other human systems [17]. The PNE education could help to change the behavior and the expectations of the patient about pain, and this change could improve the functional and psychological status of the individual who experiences pain [17].
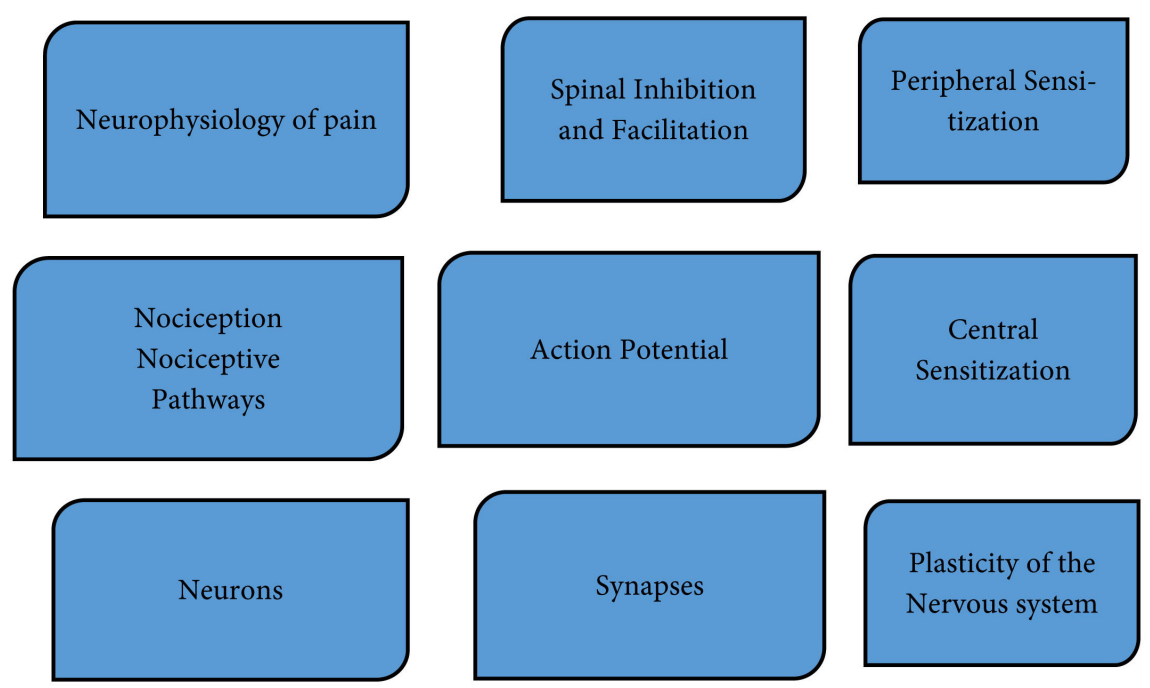

Figure 2. Basic topics in PNE. Methods of PNE. Source: https://www.physio-pedia.com. Published January 16, 2016. Accessed March 13, 2020. 
Table 2. Therapeutic interventions with this case.

\begin{tabular}{|c|c|}
\hline & Physical Therapy Interventions categories \\
\hline ESWT & $15-\mathrm{mm}$ head with 2000 pulses at each session 3 times weekly \\
\hline Manual therapy & $\begin{array}{l}\text { Joint mobilization and myofascial release techniques increase the } \\
\text { flexibility of the joint and muscles of the LL }\end{array}$ \\
\hline Stretching exercises & For lower limbs (fascia and muscles) \\
\hline Taping & Using low dye taping technique to minimize foot pronation \\
\hline Strengthening exercises & LL for weak muscles \\
\hline
\end{tabular}

\section{Outcomes}

After 12 visits, or 6 weeks of treatment, we completed a reassessment. Previous parameters were used to measure the pain and function of the foot. An assessment was completed at every session before and after treatment, and the average of the weekly scores was taken and documented in Tables 3-5.

The PSQI was used to evaluate the sleep quality level at the baseline and after the last session. At the initial evaluation, the PSQI was a 17; this number reduced minimally to a 16 at reassessment. For reference, the range of the PSQI is from 0 , meaning good sleep quality, to 21 , indicating poor sleep quality [25].

There was a noted decrease in pain in the left foot, and significant decrease to no pain in the right foot. The pre-intervention subjective function of the foot improved from $87 / 170$ on the right foot to $0 / 170$, while the function of the left foot improved from $115 / 170$ to $39 / 170$ via the FFI. It should be noted that there was still mild pain in the left foot after the prescribed 12 sessions, therefore, while physical therapy could help to reduce pain and improve the function of the feet, there was not a complete resolution of symptoms with the addition of PNE. There were slight changes in the Navicular height results of both feet by the end of the sixth week but these changes could be due to another factor such as the change of the BMI which was not reported at that stage of the study.

\section{Discussion}

The participant in this case suffered from chronic PF and had previously tried many treatment methods such as medications and traditional physical therapy. The purpose of this study was to open the door to study the potential effects of adding PNE to the conventional treatment of PF.

In this case, there were many physical causes for the patient's chronic pain. One cause of pain was spasms in the gastrocnemius and the hamstring. Moreover, the weakness of the plantar flexors caused a biomechanical disturbance of the foot which led to increased pressure on the pressure points of the medial aspect of the foot, causing continuous stress on the foot during weight bearing [9] [14].

The strengthening exercises completed at the clinic and at the patient's home were prescribed to help reduce the patient's antalgic gait pattern by increasing 
Table 3. The pain results of bilateral foot pre-treatment and post-treatment.

\begin{tabular}{cccccccc}
\hline \multicolumn{7}{c}{ VAS } \\
\hline Treatment periods & Foot & $\begin{array}{c}\text { Week 1 } \\
\text { "Baseline" }\end{array}$ & Week 2 & Week 3 & Week 4 & Week 5 & Week 6 \\
\hline \multirow{2}{*}{ Pre-intervention } & Right & 5 & 2 & 2 & 2 & 1 & 0 \\
& Left & 7 & 3 & 6 & 5 & 3 & 3 \\
\hline \multirow{2}{*}{ Post-intervention } & Right & 3 & 2 & 2 & 0 & 0 & 0 \\
& Left & 5 & 4 & 4 & 7 & 2 & 2 \\
\hline
\end{tabular}

*All numbers are on a 0 - 10 VAS scale.

Table 4. The functional results of bilateral foot pre-treatment and post-treatment.

\begin{tabular}{|c|c|c|c|c|c|c|c|}
\hline \multicolumn{8}{|c|}{ FFI } \\
\hline Treatment periods & Foot & $\begin{array}{c}\text { Week } 1 \\
\text { "Baseline" }\end{array}$ & Week 2 & Week 3 & Week 4 & Week 5 & Week 6 \\
\hline \multirow{2}{*}{ Pre-intervention } & Right & 87 & 78 & 78 & 78 & 0 & 0 \\
\hline & Left & 115 & 88 & 88 & 115 & 55 & 39 \\
\hline \multirow{2}{*}{ Post-intervention } & Right & 87 & 45 & 35 & 35 & 0 & 0 \\
\hline & Left & 105 & 87 & 87 & 88 & 52 & 31 \\
\hline
\end{tabular}

*All numbers are on a 0 - 170 FFI scale.

Table 5. Navicular height measurements.

\begin{tabular}{cccccccc}
\hline \multicolumn{7}{c}{ Navicular Drop Test } \\
\hline Treatment periods & Foot & Week 1 & Week 2 & Week 3 & Week 4 & Week 5 & Week 6 \\
\hline \multirow{2}{*}{ Pre-intervention } & Right & $11 \mathrm{~mm}$ & $11 \mathrm{~mm}$ & $10 \mathrm{~mm}$ & $11 \mathrm{~mm}$ & $11 \mathrm{~mm}$ & $10 \mathrm{~mm}$ \\
& Left & $13 \mathrm{~mm}$ & $13 \mathrm{~mm}$ & $12 \mathrm{~mm}$ & $13 \mathrm{~mm}$ & $12 \mathrm{~mm}$ & $12 \mathrm{~mm}$ \\
& Right & $11 \mathrm{~mm}$ & $10 \mathrm{~mm}$ & $10 \mathrm{~mm}$ & $10 \mathrm{~mm}$ & $11 \mathrm{~mm}$ & $10 \mathrm{~mm}$ \\
Post-intervention & Left & $13 \mathrm{~mm}$ & $13 \mathrm{~mm}$ & $12 \mathrm{~mm}$ & $13 \mathrm{~mm}$ & $12 \mathrm{~mm}$ & $12 \mathrm{~mm}$ \\
\hline
\end{tabular}

the stability of the lower limb while ambulating. This would also help to distribute body weight over the foot without concentration in a certain region, in order to reduce the risk of high-pressure areas [29] [30]. Few studies have shown the effectiveness of PNE in treating a variety of diseases and conditions [15] [16]. In this case, the patient's mental health played a significant role in producing pain, as the patient reported distress related to his family, as well as fears of solitude. We supported the patient by staying calm, and by giving him the opportunity for his nervous system to adapt to his situation and reduce the intensity of his symptoms. This support came mainly through comprehensive patient education about his pain. While using PNE, it is especially important to educate the patient on the reality of their pain and to empathize with them [14]. In addition, it is imperative to keep the patient in touch with the maintenance of his nervous system through giving recommendations such as getting enough sleep and 
working to reduce their stress levels. These factors have negative effects on the human body; they have a significant negative relationship in individuals with chronic pain due to the alteration of the chemicals in the brain and the hormones that cause mood alterations and anxiety [30]. There was continuous contact with the patient through social media by using some contact applications. Email also was used to answer any questions and also to help the patient to change some of his ideas about pain. This method of contact has been proven by Louw et al. as a way of conducting effective PNE [31]. Although the patient had a higher level of health literacy, there was a significant misunderstanding of pain knowledge and also the role of physical therapy. Although he had tried many therapeutic methods such as medications, massage, and orthotics, he could not cope with his symptoms. He had had bad experiences with physical therapy, which presented us with a challenge: every word of the PNE had to be chosen carefully to avoid the negative effects of terms such as "pain" [32]. PNE "image management" was more useful to the patient than providing stories or metaphors about the therapeutic uses of pain education [32].

A few outcome measures were used in this study in an attempt to capture objective information on how the patient perceived his pain, sleep quality and foot function. These were typically filled out before and after the treatment sessions. The data from the results of this study, as Table 2 shows, indicate a significant decrease of pain levels and an improvement in foot function, although there was still mild pain after the treatment program. The patient reported feeling satisfied with the results of his hybrid treatment. This result ties well with another systematic review done by Louw and his colleagues which shows the effectiveness of PNE in reducing pain level and stress [16]. Therefore, these results could suggest that the use of PNE in combination with conventional physical therapy treatment of PF has a positive impact by calming the nervous system and reducing pain intensity. Moreover, the self-beliefs about the participant's pain had positive changes that were observed and recorded in the evaluation records. However, it would be more beneficial to measure this change by using some outcome measures such as chronic pain self-efficacy scale (CPSS) as a good measure to detect the psychological parameter about chronic pain [33].

For that reason, this study has several limitations. For example, the sample study involved only one person; the effect of PNE should be measured with a study involving many participants comparing a control group and experimental group. Moreover, in this study, we followed the protocol of PF in one specific outpatient physical therapy clinic. There are evaluation and treatment guidelines for PF patients that are evidence-based, however, the protocol followed contained some components that should be updated to be in alignment with these guidelines. For example, the use of myofascial release is still the preferred initial intervention in this clinic despite the fact that there is little evidence supporting it as a treatment for PF [9]. On the other hand, stretching exercises for the plantar flexors and strengthening for the muscles of the lower limbs are a part of our 
protocol and are supported by CPGs of APTA [9] [29]. Low dye tape is another evidence-based intervention used in our clinic that is employed with foot orthotics [9] [11]. However, some interventions with conflicting evidence, such as dry needling, still persist in the protocols of our clinic [9]. Also, the use of ESWT is widely used with many patients, even though the effects of this intervention have only mild evidence, with no superiority over other passive modalities such as ultrasound [9]. In one study by Thomson and colleagues, ESWT showed significant benefits for treating PF [12]. Another study has demonstrated the superior effects of ESWT on corticosteroid injections for long-term pain reduction [26].

\section{Conclusion}

This case study shows the potential effects of adding PNE to conventional PT for PF treatment. Although this case did not result in complete resolution of symptoms, the patient did report improvement with symptoms and function, giving hope that this could be a valuable addition to future treatments. PNE works to help motivate the normal physiology of the nervous system and reduce the factors that could produce pain. It is recommended that more studies be done to measure the effect of PNE among people with PF and people with chronic pain with more participants than that of this case study.

\section{Conflicts of Interest}

The authors declare that there is no conflict of interest regarding the publication of this article.

\section{References}

[1] Young, C.C., Rutherford, D.S. and Niedfeldt, M.W. (2001) Treatment of Plantar Fasciitis. American Family Physician, 63, 467-474.

[2] Stecco, C., Corradin, M., Macchi, V., et al. (2013) Plantar Fascia Anatomy and Its Relationship with Achilles Tendon and Paratenon. Journal of Anatomy, 223, 665-676. https://doi.org/10.1111/joa.12111

[3] Monteagudo, M., Maceira, E., Garcia-Virto, V., et al. (2013) Chronic Plantar Fasciitis: Plantar Fasciotomy versus Gastrocnemius Recession. International Orthopaedics, 37, 1845-1850. https://doi.org/10.1007/s00264-013-2022-2

[4] Wearing, S.C., Smeathers, J.E., Urry, S.R., et al. (2006) The Pathomechanics of Plantar Fasciitis. Sports Medicine, 36, 585-611. https://doi.org/10.2165/00007256-200636070-00004

[5] Petraglia, F., Ramazzina, I. and Costantino, C. (2017) Plantar Fasciitis in Athletes: Diagnostic and Treatment Strategies. A Systematic Review. Muscles, Ligaments and Tendons Journal, 7, 107-115. https://doi.org/10.11138/mltj/2017.7.1.107

[6] Roxas, M. (2005) Plantar Fasciitis: Diagnosis and Therapeutic Considerations. Alternative Medicine Review, 10, 83-93.

[7] Thomas, M.J., Whittle, R., Menz, H.B., et al. (2019) Plantar Heel Pain in MiddleAged and Older Adults: Population Prevalence, Associations with Health Status and Lifestyle Factors, and Frequency of Healthcare Use. BMC Musculoskeletal Disord- 
ers, 20, Article No. 337. https://doi.org/10.1186/s12891-019-2718-6

[8] Van Leeuwen, K.D.B., Rogers, J., Winzenberg, T., et al. (2016) Higher Body Mass Index Is Associated with Plantar Fasciopathy/"Plantar Fasciitis": Systematic Review and Meta-Analysis of Various Clinical and Imaging Risk Factors. British Journal of Sports Medicine, 50, 972-981. https://doi.org/10.1136/bjsports-2015-094695

[9] Martin, R.L., Davenport, T.E., et al. (2014) Heel Pain-Plantar Fasciitis: Revision. JOSPT, 44, A1-A33. https://doi.org/10.2519/jospt.2014.0303

[10] Stuber, K. and Kristmanson, K. (2006) Conservative Therapy for Plantar Fasciitis: A Narrative Review of Randomized Controlled Trials. Journal of the Canadian Chiropractic Association, 50, 118-133.

[11] Newell, T., Simon, J. and Docherty, C.L. (2015) Arch-Taping Techniques for Altering Navicular Height and Plantar Pressures during Activity. Journal of Athletic Training, 50, 825-832. https://doi.org/10.4085/1062-6050-50.5.05

[12] Thomson, C.E., Crawford, F. and Murray, G.D. (2005) The Effectiveness of Extra Corporeal Shock Wave Therapy for Plantar Heel Pain: A Systematic Review and Meta-Analysis. BMC Musculoskeletal Disorders, 6, Article No. 19.ๆ https://doi.org/10.1186/1471-2474-6-19

[13] Buchanan, B.K. and Kushner, D. (2020) Plantar Fasciitis.] StatPearls Publishing (FL). https://www.ncbi.nlm.nih.gov/books/NBK431073/

[14] Fourchet, F., Kelly, L., Horobeanu, C., et al. (2015) High-Intensity Running and Plantar-Flexor Fatigability and Plantar-Pressure Distribution in Adolescent Runners. Journal of Athletic Training, 50, 117-125.

https://doi.org/10.4085/1062-6050-49.3.90

[15] Louw, A., Zimney, K., Puentedura, E.J., et al. (2016) The Efficacy of Pain Neuroscience Education on Musculoskeletal Pain: A Systematic Review of the Literature. Physiotherapy Theory and Practice, 32, 332-355. https://doi.org/10.1080/09593985.2016.1194646

[16] Louw, A., Diener, I., Butler, D.S., et al. (2011) The Effect of Neuroscience Education on Pain, Disability, Anxiety, and Stress in Chronic Musculoskeletal Pain. Archives of Physical Medicine and Rehabilitation, 92, 2041-2056.

https://doi.org/10.1016/j.apmr.2011.07.198

[17] Louw, A., Puentedura, E.J., Zimney, K., et al. (2016) Know Pain, Know Gain? A Perspective on Pain Neuroscience Education in Physical Therapy. Journal of Orthopaedic \& Sports Physical Therapy, 46, 131-134.

https://doi.org/10.2519/jospt.2016.0602

[18] Cuthbert, S.C. and Goodheart, G.J. (2007) On the Reliability and Validity of Manual Muscle Testing: A Literature Review. Chiropractic \& Osteopathy, 15, Article No. 4. https://doi.org/10.1186/1746-1340-15-4

[19] De Garceau, D., Dean, D., Requejo, S.M., et al. (2003) The Association between Diagnosis of Plantar Fasciitis and Windlass Test Results. Foot \& Ankle International, 24, 251-255. https://doi.org/10.1177/107110070302400309

[20] Spörndly-Nees, S., Dåsberg, B., Nielsen, R.O., et al. (2011) The Navicular Position test-A Reliable Measure of the Navicular Bone Position during Rest and Loading. International Journal of Sports Physical Therapy, 6, 199-205.

[21] Barouk, P. and Barouk, L.S. (2014) Clinical Diagnosis of Gastrocnemius Tightness. Foot and Ankle Clinics, 19, 659-667. https://doi.org/10.1016/j.fcl.2014.08.004

[22] SooHoo, N.F., Samimi, D.B., Vyas, R.M., et al. (2006) Evaluation of the Validity of the Foot Function Index in Measuring Outcomes in Patients with Foot and Ankle Disorders. Foot \& Ankle International, 27, 38-42. 
https://doi.org/10.1177/107110070602700107

[23] OrthoToolKit (2020) Foot Function Index. http://www.orthotoolkit.com/ffi/

[24] Venditto, T., Tognolo, L., Rizzo, R.S., et al. (2015) 17-Italian Foot Function Index with Numerical Rating Scale: Development, Reliability, and Validity of a Modified Version of the Original Foot Function Index. The Foot, 25, 12-18.

https://doi.org/10.1016/j.foot.2014.09.004

[25] Ayearst, L.E., Harsanyi, Z. and Michalko, K.J. (2012) The Pain and Sleep Questionnaire Three-Item Index (PSQ-3): A Reliable and Valid Measure of the Impact of Pain on Sleep in Chronic Nonmalignant Pain of Various Etiologies. Pain Research and Management, 17, Article ID: 635967. https://doi.org/10.1155/2012/635967

[26] Liu, Y., Liu, J., Liu, G., et al. (2019) Comparison of Efficacy of Shock-Wave Therapy versus Corticosteroids in Plantar Fasciitis: A Meta-Analysis of Randomized Controlled Trials. Archives of Orthopaedic and Trauma Surgery, 139, 529-536.

https://doi.org/10.1007/s00402-018-3071-1

[27] Brantingham, J.W., Globe, G., Pollard, H., et al. (2009) Manipulative Therapy for Lower Extremity Conditions: Expansion of Literature Review. Journal of Manipulative and Physiological Therapeutics, 32, 53-71. https://doi.org/10.1016/j.jmpt.2008.09.013

[28] Ajimsha, M.S., Binsu, D. and Chithr, S. (2014) Effectiveness of Myofascial Release in the Management of Plantar Heel Pain: A Randomized Controlled Trial. The Foot, 24, 66-71. https://doi.org/10.1016/j.foot.2014.03.005

[29] Dos Santos, B., Corrêa, L.A., Santos, L.T., et al. (2016) Combination of Hip Strengthening and Manipulative Therapy for the Treatment of Plantar Fasciitis: A Case Report. Journal of Chiropractic Medicine, 15, 310-313. https://doi.org/10.1016/j.jcm.2016.08.001

[30] Chang, B.C., Liu, D.H., Chang, J.L., et al. (2014) Plantar Pressure Analysis of Accommodative Insole in Older People with Metatarsalgia. Gait \& Posture, 39, 449 454. https://doi.org/10.1016/j.gaitpost.2013.08.027

[31] Louw, A. and Puentedura, E.J. (2014) Therapeutic Neuroscience Education, Pain, Physiotherapy and the Pain Neuromatrix. International Journal of Health Sciences, 2, 33-45. https://doi.org/10.15640/ijhs.v2n3a4

[32] Louw, A., Puentedura, E.J., Diener, I., et al. (2019) Pain Neuroscience Education: Which Pain Neuroscience Education Metaphor Worked Best? South African Journal of Physiotherapy, 75, 468-475. https://doi.org/10.4102/sajp.v75i1.1329

[33] Rondon-Ramos, A., Martinez-Calderon, J., Diaz-Cerrillo, J.L., Rivas-Ruiz, F., ArizaHurtado, G.R., Clavero-Cano, S. and Luque-Suarez, A. (2020) Pain Neuroscience Education plus Usual Care Is More Effective than Usual Care Alone to Improve Self-Efficacy Beliefs in People with Chronic Musculoskeletal Pain: A Non-Randomized Controlled Trial. Journal of Clinical Medicine, 9, 2195.

https://doi.org/10.3390/jcm9072195 